\title{
EchoGéo
}

$43 \mid 2018$

Processus de gouvernance dans les territoires ruraux

\section{Les sociétés locales rurales et le développement des gîtes dans la mégalopole de Shanghai}

Gouvernance rurale et prestations de services touristiques

\section{Emmanuel Véron}

\section{OpenEdition}

\section{Journals}

Electronic version

URL: https://journals.openedition.org/echogeo/15209

DOI: 10.4000/echogeo.15209

ISSN: 1963-1197

Publisher

Pôle de recherche pour l'organisation et la diffusion de l'information géographique (CNRS UMR 8586)

\section{Electronic reference}

Emmanuel Véron, "Les sociétés locales rurales et le développement des gîtes dans la mégalopole de Shanghai", EchoGéo [Online], 43 | 2018, Online since 22 March 2018, connection on 23 August 2021. URL: http://journals.openedition.org/echogeo/15209; DOI: https://doi.org/10.4000/echogeo.15209

This text was automatically generated on 23 August 2021.

EchoGéo est mis à disposition selon les termes de la licence Creative Commons Attribution - Pas d'Utilisation Commerciale - Pas de Modification 4.0 International (CC BY-NC-ND) 


\section{Les sociétés locales rurales et le développement des gîtes dans la mégalopole de Shanghai}

Gouvernance rurale et prestations de services touristiques

\section{Emmanuel Véron}

\section{Introduction}

1 En avril 2017, un communiqué de l'agence de presse chinoise Xinhua ${ }^{1}$ relayait les chiffres du Ministère de l'Agriculture sur les revenus du tourisme rural en Chine pour l'année 2016, qui s'élevaient à plus de 570 milliards de yuans (environ 83 milliards de dollars). Avec environ 2,1 milliards de voyages effectués à la campagne en 2016, le secteur aurait créé des emplois pour 6,72 millions de ruraux. Ces chiffres témoignent à eux seuls de l'important développement du secteur touristique dans les campagnes chinoises.

2 Cet article vise à analyser le rôle des sociétés locales dans le développement touristique des lieux, en particulier des villages touristiques dans le delta du Yangzi (Chine) ${ }^{2}$. La diffusion et le développement du tourisme révèlent un profond renouvellement des dynamiques rurales et sociales depuis l'ouverture économique du territoire et de la société chinoise (Véron, 2016). Si le processus de développement des lieux touristiques est principalement issu d'un effet du haut vers le bas, par des politiques d'intégration verticale, la dimension horizontale n'en demeure pas moins importante. Nous montrerons que les sociétés locales sont des acteurs de premier plan dans un double processus de démarrage et d'adaptation.

3 C'est dans les transformations sociales, politiques et économiques des villages que le tourisme et l'accueil à la ferme puisent leurs origines. La question de la participation des communautés rurales au développement économique des localités par le tourisme est essentielle. Les mutations post-maoïstes du cadre villageois ont réintroduit la pluriactivité des populations paysannes. Ces observations s'inscrivent dans un contexte 
scientifique plus large, celui de la gouvernance locale en tourisme et les jeux de pouvoir entre plusieurs acteurs (Hall, 2003; Church et Cole, 2006 ; Beaumont et Dredge, 2008 ; Bramwell et Lane, 2011). L'insertion des ruraux dans le tourisme est intimement liée au contexte politique et économique local. Le développement du tourisme est un ressort important pour le renouvellement des rapports de force entre différents acteurs locaux et extra-locaux (communautés, professionnels du tourisme et autorités).

4 À l'instar d'observations des campagnes dans les pays occidentaux, la fonction tertiaire (tourisme) prend appui sur deux décennies de multifonctionnalités des espaces ruraux, en particulier ceux dont la distance à la ville permet la complémentarité de différentes formes de revenus. Les activités touristiques sont de plus en plus perçues comme un moyen de diversifier les formes de revenus dans un contexte où la paysannerie est en profonde mutation, du fait d'une rentabilité trop faible des agricultures. Ce texte montrera les diverses formes de gouvernance rurale intégrant des communautés rurales dans le processus de modernisation des espaces ruraux et d'intégration villecampagne.

5 Nous examinerons les conditions de l'émergence de la participation des sociétés locales au tourisme, puis nous distinguerons les différentes formes d'organisation collective en lien avec les autorités locales rurales. Nous avons privilégié une méthodologie qualitative basée sur des entretiens/discussions avec des responsables de gîtes et des cellules locales du Parti. L'étude de deux villages touristiques dans la mégalopole (mégalopolis) delta du Yangzi permet de rendre compte des modes récents de gouvernance rurale.

\section{Dynamiques rurales et diversification des revenus non agricoles : émergence du secteur des services touristiques}

6 Le lancement des réformes à la fin des années 1970 a amorcé une période de renouvellement des processus de gouvernance des campagnes chinoise. La décollectivisation (début des années 1980) démantèle les communes populaires (renmin gongshe). Ces dernières se transforment en cantons et bourgs. Par ailleurs, les échelons inférieurs (brigades de productions - shengchan dadui et équipes de production shengchan dui) changent de statut. Les brigades se transforment en villages administratifs (xingzheng cun), tandis que les villages (ziran cun - des hameaux) correspondent aux anciennes équipes de production. Ces transformations recomposent les systèmes productifs agricoles et industriels dans les campagnes, et modifient la répartition du pouvoir, faisant du comité de villageois (cunmin weiyuanhui) ${ }^{3}$ et du Parti communiste, l'organe du pouvoir officiel au sein du village.

7 Ces transformations politiques sociales conduisent à une réorganisation des sociétés paysannes et rurales. Le comité villageois est une institution très encadrée. La cellule locale du Parti exerce un contrôle sur l'ensemble des activités du comité. Les communautés locales gèrent désormais «leurs propres affaires » à travers l'introduction d'élections. Les villages administratifs ne relèvent pas de l'État, mais incarnent un niveau d'autonomie (zizhi) (Charon, 2013).

8 Parallèlement aux mutations politiques, les réformes signifient une nouvelle liberté pour les ménages paysans et non paysans résidant dans les campagnes, qui leur permet 
d'accroître leurs revenus, mais aussi de diversifier le spectre des emplois permanents ou non. Ainsi les activités annexes au travail agricole augmentent-elles de façon importante le revenu non agricole. L'économie (re)devient marchande dans les campagnes, provoquant de nouvelles occasions parfois inédites de s'enrichir en particulier en articulation avec la ville (Sanjuan, 2011). La pluriactivité (re)devient une norme faisant du district une base économique et commerciale, auxquels les villages sont nouvellement articulés (Aubert, 2005).

9 Aussi, les migrations de la campagne vers la ville ont pu permettre de stimuler des revenus suffisants (Démurger et $\mathrm{Xu}, 2011 \mathrm{~b}$ ), de développer des compétences nouvelles et d'élargir le réseau de relations pour introduire au village des activités économiques nouvelles, souvent orientées vers une activité marchande. Isabelle Thireau indique que « les ressources mobilisées pour justifier l'activité marchande sont rarement liées à un savoir-faire économique, mais sont plutôt ancrées dans la sphère sociale ou politique " (Thireau et Hua, 2002, p. 125).

10 La spatialisation de l'industrialisation rurale est hétérogène selon les régions. Les entités littorales (Shanghai, Jiangsu et Zhejiang) sont davantage marquées par le processus industriel que la province de l'Anhui, encore rurale agricole (Aubert, 2005), dont le cortège des migrants paysans est particulièrement important. Les entreprises des bourgs et des cantons (EBC) ont permis l'enrichissement des ménages ruraux depuis le milieu des années 1980 et ont polarisé le travail non agricole durant la même période. Yang (2005) démontre qu'au début des années 2000, 96,6\% des entreprises rurales sont des entreprises privées (dont les propriétaires ont des liens étroits avec les responsables locaux). Les deux décennies de développement rural dû aux changements structurels induits par les $\mathrm{EBC}$ ont réuni les conditions nécessaires et favorables pour que les communautés rurales (paysannes ou non) accumulent un capital (social, technique et économique) suffisant pour initier de nouvelles activités.

Depuis le milieu des années 2000, les coopératives (hezuoshe) constituent de nouvelles entités économiques et sociales très souples (en termes de propriété et de gestion) et polarisant aisément des capitaux. Ces coopératives sont spécialisées (zhuanye) par branche économique. Les systèmes coopératifs par part (gufen hezuozhi) (parfois appelées coopératives par part - gufen hezuoshe) correspondent à un mélange de propriétés collectives et de parts de gestionnaires (Ren, 2011). Ces coopératives rassemblent des individus d'une même communauté villageoise réunis afin d'assurer la pérennité des activités locales autour de la terre ou d'autres domaines. La coopérative est intimement articulée au comité de villageois et à la cellule du Parti. Souvent, les actionnaires et/ou leaders de la coopérative ont aussi un rôle dans le comité ou le Parti en tant que cadres.

12 La participation (canyu) des communautés rurales, via des coopératives ou d'autres formes d'organisations collectives, autour d'un leader dans le processus touristique polarise l'intérêt des cadres villageois, des responsables des cellules locales du Parti et les individus eux-mêmes. Par ailleurs, cette forme de tourisme où les foyers paysans forment la base du produit touristique (accueil, hébergement, restauration voire activités d'animation) retient l'attention des voyagistes. En effet, l'intégration des communautés rurales dans le projet touristique à l'échelle du village contraste avec les sites touristiques gérés par des compagnies spécialisées, des investisseurs et des acteurs gouvernementaux. C'est bien l'idée d'un développement par le bas (bottom-up) qui prend forme et la relance des débats sur le développement économique, social, voire 
politique des populations rurales (en particulier paysannes) (Barthélemy, et al., 2004 ; Rodriguez et al., 2011; Wu et al., 2015). Cette forme de tourisme répondrait partiellement à la crise agricole, à la modernisation rurale, aux migrations des populations les plus démunies, et inclurait localement les populations dans une démarche nouvelle.

13 L'activité touristique est analysée comme une stratégie des familles ${ }^{4}$ ne trouvant plus dans l'agriculture, mais aussi dans les autres sources de revenus non agricoles, des activités rentables. Le complément puis, la supplantation ou plus exactement la domination des recettes touristiques permet de maintenir et d'améliorer l'agriculture, d'augmenter son niveau de vie (achats de véhicules, équipements modernisés du foyer, finances supplémentaires pour la famille) et d'accéder à un statut nouveau de prestataire. C'est pourquoi le gîte peut servir parfois à l'affirmation du statut social de l'individu et de vitrine pour l'exploitation touristique.

\section{Sociétés locales et processus touristique : une organisation collective par le bas?}

14 Les chercheurs chinois (He et al, 2004 ; Zheng et Zhong, 2004; Shao, 2007 ; Hu, 2007 et 2009 ; Su, 2011) ont décrit six catégories différentes d'organisation des sociétés locales incluses dans le processus touristique dans les campagnes. Ceci offre un cadre privilégié d'un développement local par le bas, corrélé à des politiques nationales de développement rural et touristique.

\section{L'initiative commerciale individuelle}

15 Représentée par le nom générique de nongjiale ${ }^{5}$, elle est la base de la relation commerciale et de la prestation touristique dans les campagnes (Véron, 2013). Cette catégorie (informelle) est articulée à des lieux de tourisme gérés et développés par les gouvernements locaux et des entreprises privées (hauts lieux, parcs, villages dont la fréquentation touristique est forte, soit plusieurs centaines de milliers de touristes nationaux et internationaux par an). La prestation reste simple et peu sophistiquée : couvert, éventuellement point de vente, parfois hébergement (avec un niveau de confort minimum). L'individu est indépendant de toutes normes institutionnalisées à l'échelle d'un lieu touristique. L'activité commerciale n'est pas l'objet d'un enregistrement au comité du village ou de la cellule locale du Parti. Malgré la proximité d'un lieu touristique, le paysan ne participe pas administrativement parlant aux recettes touristiques du village. L'activité est déconnectée du processus réglementaire (tarif, hygiène, normes) tout en étant articulée au lieu. Les autorités connaissent l'ampleur des activités, mais n'interviennent qu'en cas d'excès. Les paysans se voient alors soumis à des taxes supplémentaires voire à des intimidations.

La logique commerciale s'inscrit dans une complémentarité d'activités et des revenus de l'agriculture. L'investissement financier dans l'activité touristique est réduit au minimum (équipements de cuisine et de table; panneaux de décoration et de signalisation de la ferme sur le bord de route). Les revenus générés par l'activité touristique ponctuelle contribuent à la modernisation du bâti, l'achat d'engrais, de matériels agricoles et éventuellement d'un véhicule d'occasion. L'activité marchande ne supplante pas l'activité agricole ou d'autres activités. 


\section{Les fermes individuelles} de participation au processus touristique plus élaborée que la forme précédente. Les statistiques chinoises distinguent les entreprises individuelles ou getihu (sept salariés maximum) des entreprises privées (plus de sept salariés). La relation marchande est d'un niveau plus élevé que la simple démarche isolée d'un individu. Ici, la famille joue le rôle majeur dans l'accueil touristique et l'éventuelle animation. La complémentarité avec l'agriculture est déterminante. La transformation de la ferme avec l'aménagement d'une salle de restauration, de chambres et un parking nécessitent des fonds recueillis à l'aide des revenus touristiques et de l'entraide entre les membres d'une même famille ${ }^{6}$. Le travail et les tâches sont répartis entre les différents membres de la cellule familiale rurale. L'entreprise familiale individuelle et ses activités marchandes sont enregistrées auprès des instances politiques et de l'administration du village (comité de villageois et cellule du Parti). Le canton et le bourg ont également connaissance des activités des familles (en particulier lorsque l'activité touristique concerne plusieurs foyers).

\section{La forme de la coopération entre plusieurs foyers ruraux (ou familles paysannes)}

coopération constitue le troisième type de participation au processus touristique. Cette forme diffère des deux précédentes par la spécialisation de la famille ou du groupe de familles dans un produit touristique (yi hu yi zhuanye xiangcun lüyou). L'objectif de cette forme collective de participation et d'organisation du processus touristique vise à moderniser et augmenter les revenus agricoles en s'appuyant sur le développement touristique.

Les activités sont connues des instances villageoises et sont plutôt bien perçues. L'augmentation $\mathrm{du}$ niveau de vie et l'organisation collective participent au développement rural en procurant des richesses aux gouvernements locaux, souvent en quête de ressources pour les finances locales (retraites, infrastructures, équipements et budget global). Le budget des revenus du tourisme participe à la vitalité de l'économie agricole du village en introduisant une sorte de complémentarité entre des familles orientées vers l'accueil à la ferme et des foyers paysans maintenus dans l'agriculture.

\section{Synergie entre entreprise privée et paysans}

La quatrième forme correspond à une synergie entre une entreprise privée et des paysans pour développer un produit touristique à l'échelle d'un village (gongsi jia nonghu). Cette forme est initiée par les autorités locales (soit le comité du village, soit la cellule du Parti, soit les deux). Ces dernières sont en lien avec une entreprise privée spécialisée dans le tourisme qui intervient dans le développement du produit touristique au sein du village. La compagnie loue par bail les terres du village afin de développer les infrastructures touristiques. Les foyers ruraux sont associés au projet. Ils suivent les orientations suggérées par la compagnie à la fois en termes de gestion des gîtes et chambres d'hôtes, de tarification des repas et des nuitées, mais aussi pour 
l'animation du village (jeux, attractions, festivals, guides). Les professionnels du tourisme apportent l'aide technique, les normes et les contacts avec les voyagistes afin de rentabiliser le village devenu lieu touristique. Dans ce cadre, les familles paysannes augmentent leurs revenus. Cependant les niveaux de dépendance aux professionnels du tourisme et aux autorités locales sont accrus. L'aide apportée par l'entreprise est compensée par une captation des recettes touristiques.

\section{Associations paysannes, entreprise privée et paysans}

21 La cinquième et avant-dernière forme correspond à une synergie entre une entreprise, une association et des paysans (gongsi jia xiehui jia nonghu). Les paysans se réunissent dans une association (xiehui) représentée par le comité de villageois. Chaque foyer est un élément actif au sein de l'association, dans laquelle il est représenté par un membre de la famille paysanne (le plus souvent le père, chef de famille).

L'entreprise apporte des savoir-faire et des financements. Elle est en constante relation avec les paysans via l'association pour l'établissement des tarifs, des normes et des standards. Les paysans sont employés par l'entreprise comme guides, animateurs, pour la billetterie et l'entretien du site. L'entreprise et l'association s'accordent pour la redistribution des recettes en parts égales à l'ensemble des foyers paysans. L'entreprise est en lien avec les professionnels du tourisme afin d'attirer la clientèle.

Un nouveau schéma économique institutionnel apparaît. Chaque acteur a un rôle déterminé et cloisonné. Les autorités locales sont à l'origine d'un tel schéma de développement, s'assurant ainsi des recettes non négligeables pour la modernisation de la communauté et des infrastructures villageoises. 


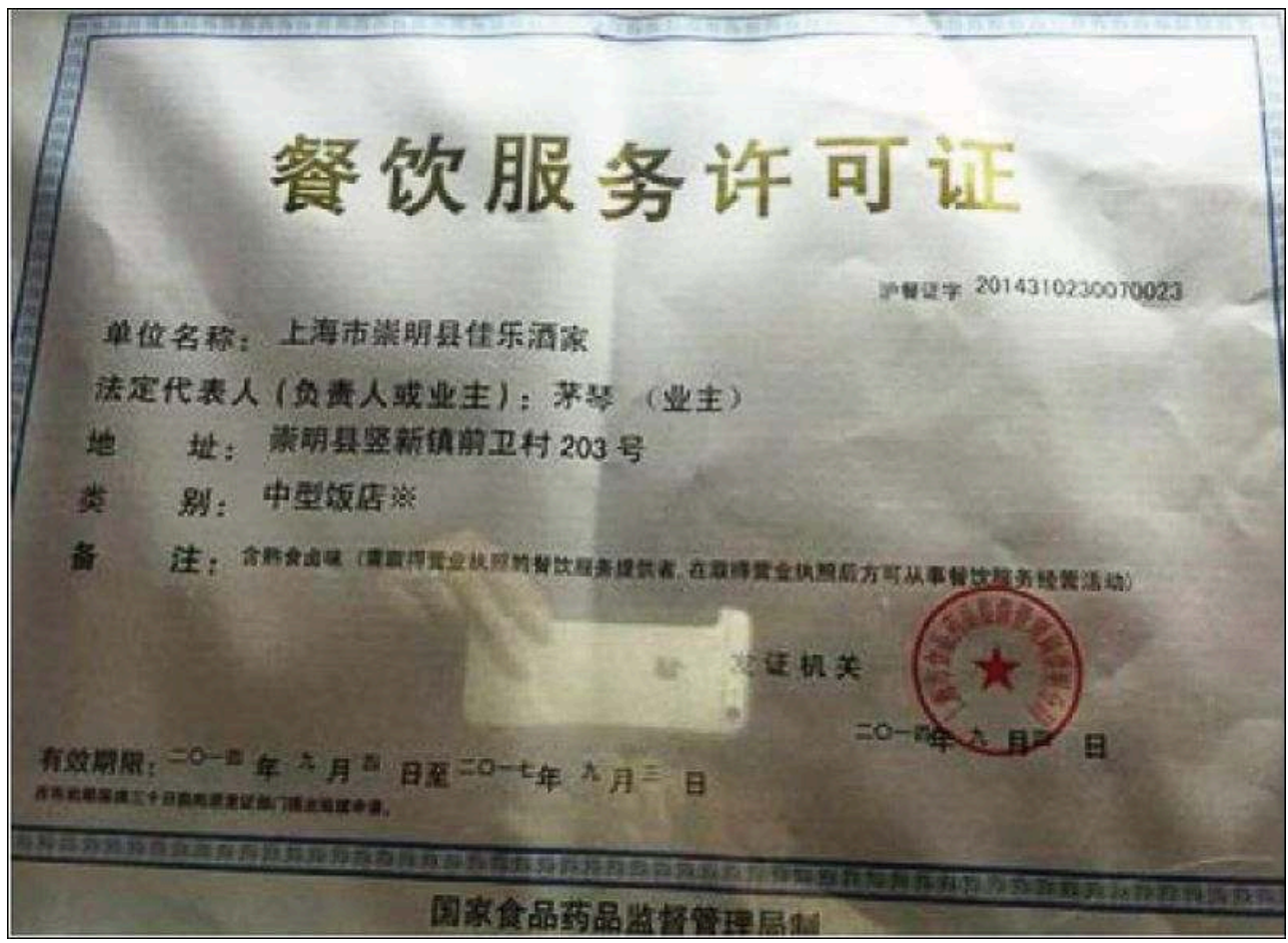

Le certificat officiel de restauration est remis par les autorités locales (comité de villageois et cellule du PCC). Chaque restaurant et chaque gîte (ici dans le village de Quianwei - Shanghai) doit être enregistré et certifié d'une autorisation d'activités. Le suivi et les normes de mise en service sont supervisés par les autorités du bourg et du district rural (sinon de la municipalité) œuvrant dans les affaires commerciales et dans les services d'hygiène. Le propriétaire se voit attribuer un numéro d'entreprise (en haut à droite de l'image) et une date de mise en service. Le développement des activités touristiques est inscrit dans un système de normes.

\section{Gouvernements locaux, entreprise privée et organisation paysanne}

La sixième et dernière forme correspond à la participation active des gouvernements locaux et centraux (Pékin) dans le développement touristique d'un lieu (d'un village dans la majeure partie des cas). Par conséquent, les lieux ont une capacité d'accueil et une taille supérieures aux formes décrites précédemment. Les lieux font l'objet d'une transformation complète pour et par le tourisme.

Les gouvernements locaux réquisitionnent les terres des paysans à l'échelle d'un hameau voire de l'ensemble d'un village administratif. Puis, le gouvernement s'organise afin d'employer l'ensemble des communautés rurales dans les entreprises du site touristique. Par ailleurs, le comité de villageois et la cellule du Parti en lien avec le canton et le bourg (comité et Parti) «ouvrent» le village aux investisseurs et entrepreneurs pour développer le site. Ces derniers acteurs sont susceptibles de prendre part aux changements structurels de l'emploi rural en employant une partie des communautés paysannes. Enfin, les paysans peuvent eux-mêmes créer leur propre entreprise employant à leur tour une main-d'œuvre locale. Cette forme renouvelle profondément le marché et la structuration de l'emploi. La contractualisation du travail (avec un système de salaire mensuel et des primes annuelles) modifie les relations à l'argent et la gestion des budgets des familles et des individus. 
Illustration 2 - Réunion de la cellule du PCC : gestion et orientation de l'activité économique des gîtes

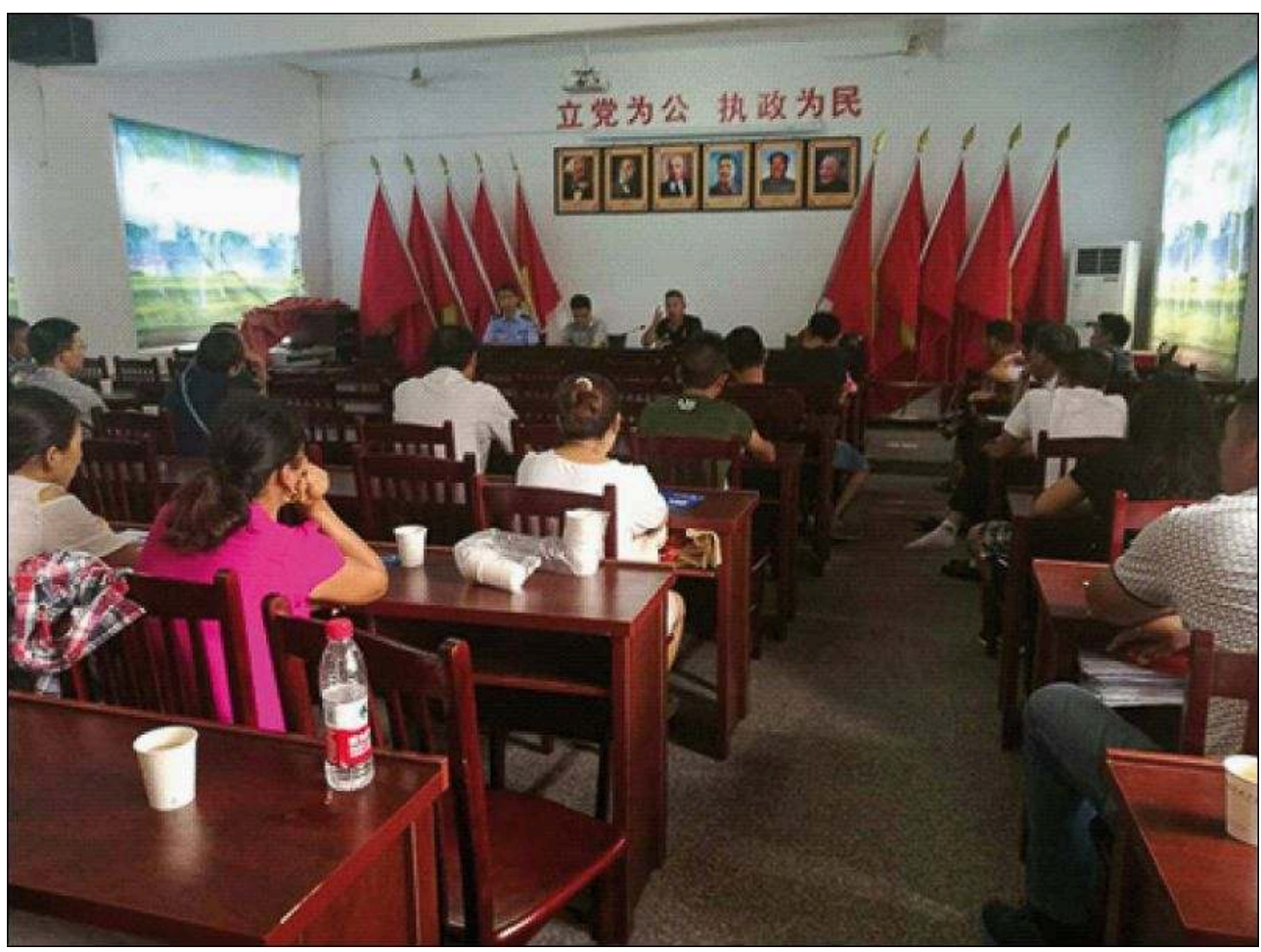

L'objet de la réunion de la cellule du Parti communiste du village de Shuikou en août 2016 est d'opérer un suivi de la gestion du produit touristique rural dans ce village. Les membres du Parti sont présents, ainsi que quelques responsables de gîtes invités. Les réunions de ce type sont mensuelles et montrent bien l'intervention et le suivi du Parti dans le développement touristique du village.

Source : Site internet de la cellule du PCC du village de Shuikou : http://shuikoudq.com/content/? 2323.html

La gestion du gîte a évolué au cours de la dernière décennie. L'introduction ( $1^{\text {er }}$ juillet 2007) d'une nouvelle loi d'encadrement des coopératives spécialisées dans les campagnes va structurer les communautés rurales évoluant dans le tourisme (Hu, 2009) d'une façon nouvelle et renforcée. Par conséquent, les deux dernières formes décrites plus haut vont être l'objet d'une nouvelle gouvernance répartie entre quatre types distincts.

- Le centre de services touristiques des gîtes (nongjiale lüyou fuwu zhongxin) d'initiative gouvernementale aménage, choisit et gère le site touristique. Ce type est opératoire à l'échelon non pas du village, mais du bourg ou du canton, pouvant ainsi fonctionner dans plusieurs villages administratifs.

- L'association des gites (nongjiale xiehui) vise à regrouper les paysans en association via le gouvernement local (comité de villageois et Parti communiste) pour la gestion des fermes touristiques. C'est la forme la plus répandue dans la gestion des villages transformés par le tourisme.

- Les Sociétés de services touristiques (lüyou fuwu gongsi) regroupent les fermes d'un ou plusieurs hameaux et gèrent la "touristification» de ce qui, désormais, est un site touristique important (tant dans sa fréquentation touristique que par sa capacité d'accueil).

Les coopératives des gîtes (nongjiale lianheshe) sont recomposées après l'adoption de la loi de 2007 (anciennement coopératives spécialisées - zhuanye hezuoshe zuzhi). Elles émanent directement de la législation nationale sur la spécialisation des paysans en 
coopération (zhonghua renmin gongheguo nongmin zhuanye hezuoshe fa) de 2007. La coopérative des gîtes appartient à l'organisation économique paysanne, elle est constituée de cinq membres minimum. Le représentant de chaque organisation participe aux réunions et activités du comité villageois, il est parfois membre de la cellule du Parti locale.

Illustration 3 - Diversité des biens touristiques : gîtes et restaurants

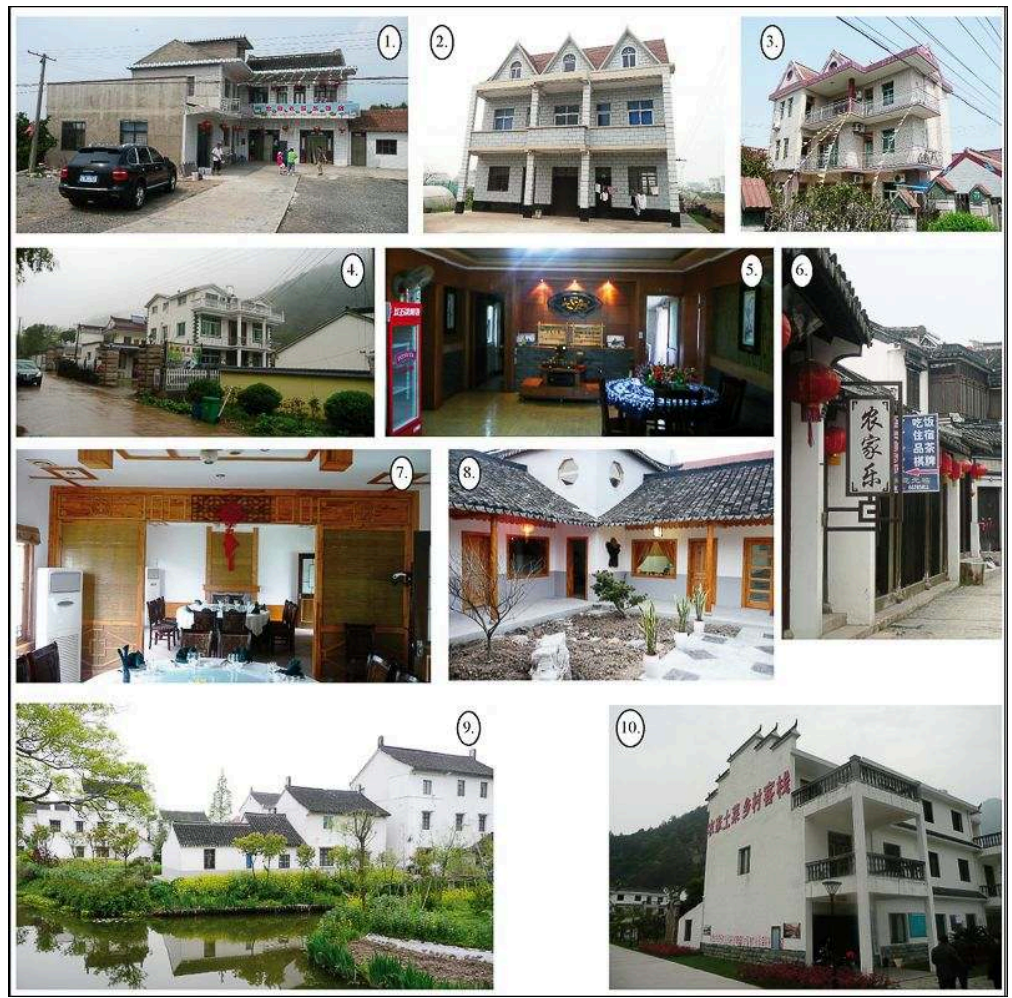

1 : restaurant en démarche individuelle à proximité du site de Xishashidi (Chongming - Shanghai), juillet $2012 ; 2$ : corps de ferme d'un paysan proposant restauration et hébergement dans une démarche individuelle (Pudong - Shanghai), avril $2009 ; 3$ : gîte sur 3 niveaux dans le village de Quianwei en association (Chongming), juin 2012; 4 : gites dans le village de Guzhu sur 3 niveaux (Changxing - Zhejiang) en association, avril $2010 ; 5$ : salle de réception d'un gîte dans le village de Shuikou (Changxing - Zhejiang) en association, , avril $2010 ; 6$ : gîte et restaurant en association dans le village de Lüxiang (lac Tai - Jiangsu), juin 2012; 7 : salle de restaurant dans la ferme reconstituée de Quiansheng (Nankin - Jiangsu), avril $2010 ; 8$ : jardin et restaurant du site de Sanyuan en coopérative (Jiansan - Shanghai), mars $2010 ; 9$ : gîtes et restaurants en gestion par la compagnie Jingjiang (Jianshan -Shanghai), avril $2009 ; 10$ : gites et restaurants dans le district de Xianju (Zhejiang) en coopérative, mai 2012.

Auteur : E. Véron.

\section{Les villages de Shuikou/Guzhu (Zhejiang), exemples de modernisation par le tourisme}

L'étude de cas permet d'appréhender les relations établies entre les différents acteurs en jeu dans le développement touristique d'un village, permettant un renouvellement original de la gouvernance rurale. Nous avons privilégié une méthodologie qualitative basée sur une série d'entretiens et de discussions avec les responsables du Parti local et plusieurs responsables de gîtes des deux villages. Plusieurs responsables nous ont été présentés dans un cadre officiel. Nous faisons le choix d'expliciter la trajectoire 
ascendante d'un individu en lien avec les autorités locales et présenté comme une réussite au sein de la zone rurale. Cette trajectoire ne doit pas être généralisée à l'ensemble de ce contexte rural, néanmoins elle rend compte de la dynamique économique locale.

Illustration 4 - La distribution des lieux de l'étude dans le delta du Yangzi

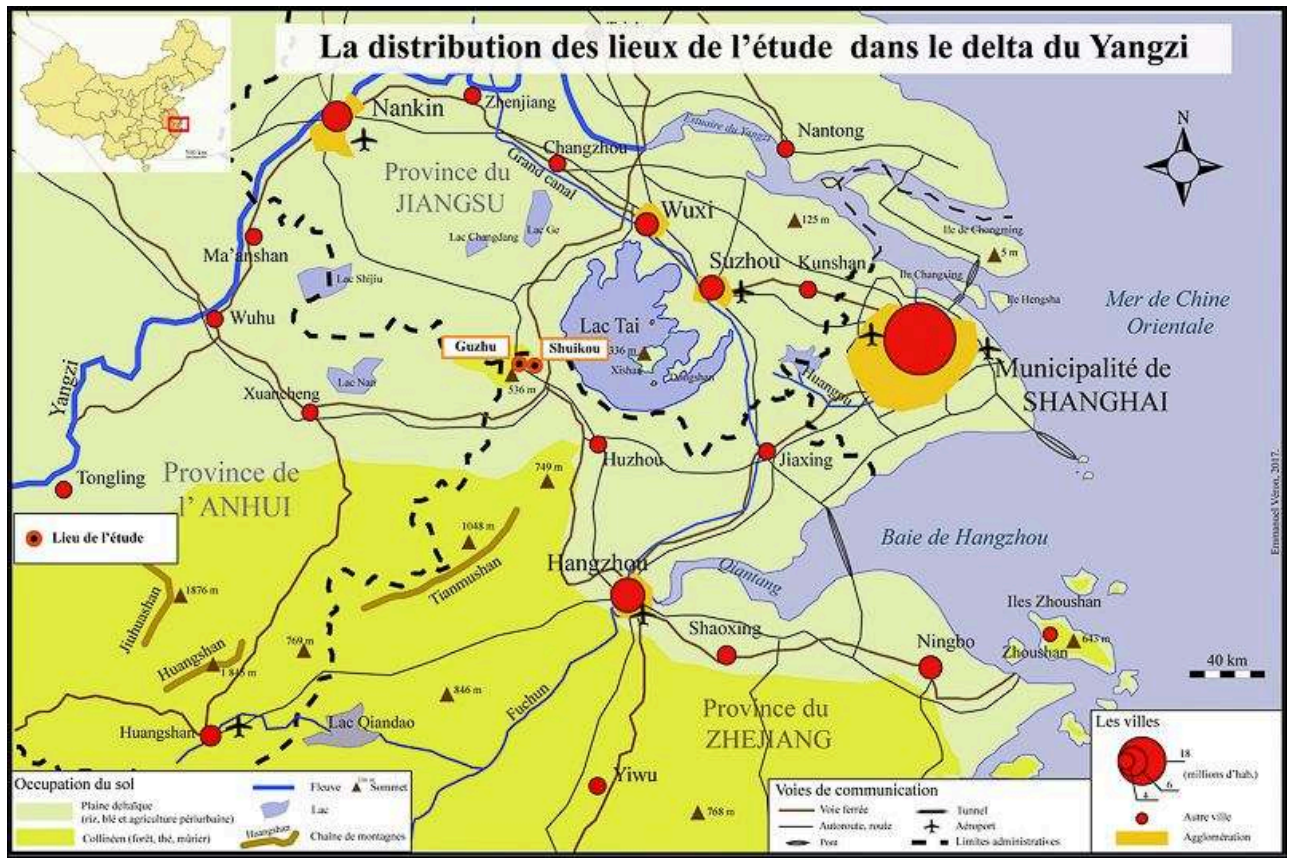

Auteur : E. Véron, 2017.

Localisés en fond de vallée à une vingtaine de kilomètres du lac Tai, les villages de Guzhu (2 567 habitants) et Shuikou (3 818 habitants) dépendent du canton de Shuikou, dans le district rural de Changxing (municipalité de Huzhou), au nord de la province du Zhejiang.

\section{Le développement local des villages de Shuikou et Guzhu dans un espace rural en périphérie de la mégalopole}

Ces deux villages adjacents sont aujourd'hui l'objet d'un développement touristique important associant villages muséifiés, espace naturel des montagnes de Guzhu et espaces cultuels à l'instar du mausolée de Lu Yu et de temples bouddhistes. Le tourisme est initié dans le milieu des années 1990. Quelques paysans aménagent un espace d'accueil pour les citadins afin d'offrir un repas et des points de vente directe de thés de la région. Dès 2000, les gouvernements du district et de la municipalité de Huzhou investissent dans la rénovation des infrastructures routières pour accroître le tourisme dans les villages. Nos observations (entre avril et octobre 2012) nous ont permis de constater que la sylviculture était présente dans l'environnement immédiat des villages de Shuikou et de Guzhu. Un arbre peut se vendre jusqu'à 40000 yuans l'unité. L'utilisation des arbres pour le développement et l'aménagement urbain est importante, surtout dans un contexte de "ville verte», de promotion de développement durable. L'arbre est destiné à quatre usages: les parcs urbains et périurbains ou autre espace public, la voirie (route et autoroute), les espaces désignés 
comme « écologiques » et plus ponctuellement les villas, grands hôtels. Les plantations d'arbres sont contemporaines du développement touristique accru des villages après 2006. Des parcs forestiers en exploitation sont destinés à la vente pour l'aménagement, tandis que des parcelles arboricoles de jujubiers sont destinées au tourisme.

31 Aussi, les paysans sont-ils de plus en plus nombreux à offrir des formules de restauration et quelques formules d'hébergement pour la clientèle de Hangzhou et de Shanghai essentiellement ${ }^{7}$ (respectivement à $1 \mathrm{~h} 30$ et $2 \mathrm{~h} 30$ de transport par l'autoroute).

La région est connue pour ses cultures de thé, mais aussi pour l'histoire du thé en Chine. En effet, le maître du thé $\mathrm{Lu} \mathrm{Yu} \mathrm{(733-804)} \mathrm{aurait} \mathrm{rédigé} \mathrm{le} \mathrm{Classique} \mathrm{du} \mathrm{thé} \mathrm{(chajing)}$ dans cette région. Les autorités locales en lien avec la province du Zhejiang « réaniment » cette histoire locale et nationale en investissant dans la construction d'un mausolée à la mémoire du maître du thé. De profonds changements interviennent donc au milieu des années 2000. Les plans du mausolée (à l'aide d'investisseurs extérieurs au village) accolent à un temple préexistant un monumental mausolée, des galeries, un musée et un espace de repos pour la consommation du thé. L'édifice voit le jour à la fin de l'année 2009.

Par ailleurs, le village est l'objet d'une coopération avec la société privée sinoaméricaine Seven Cups pour la production de thé. En effet, Mme Zhu résidant en Arizona a développé des contacts avec une dizaine de familles du village afin de s'approvisionner en thé qu'elle exporte vers les États-Unis. Aussi, cette compagnie opère dans le secteur du tourisme avec d'autres villages en Chine (notamment dans la province du Yunnan) $)^{8}$.

34 Parallèlement, la fréquentation touristique ne cesse de croître. Les paysans se sont organisés au milieu des années 2000 en association pour proposer un cadre standardisé à l'offre touristique: tarifs des nuitées, des repas, hygiène et éventuelles activités d'animation. À partir de 2010, les certifications du site, la diversification et l'implication des acteurs locaux et extra-locaux suivent une progression sans précédent. Le village connait une augmentation de la population, de la modernisation des infrastructures et du nombre de touristes par année (300 000 visiteurs en 2006 et près de 2 millions de visiteurs en 2012 selon les autorités locales). 
Illustration 5 - Le village de Shuikou : mutations spatiales et développement local

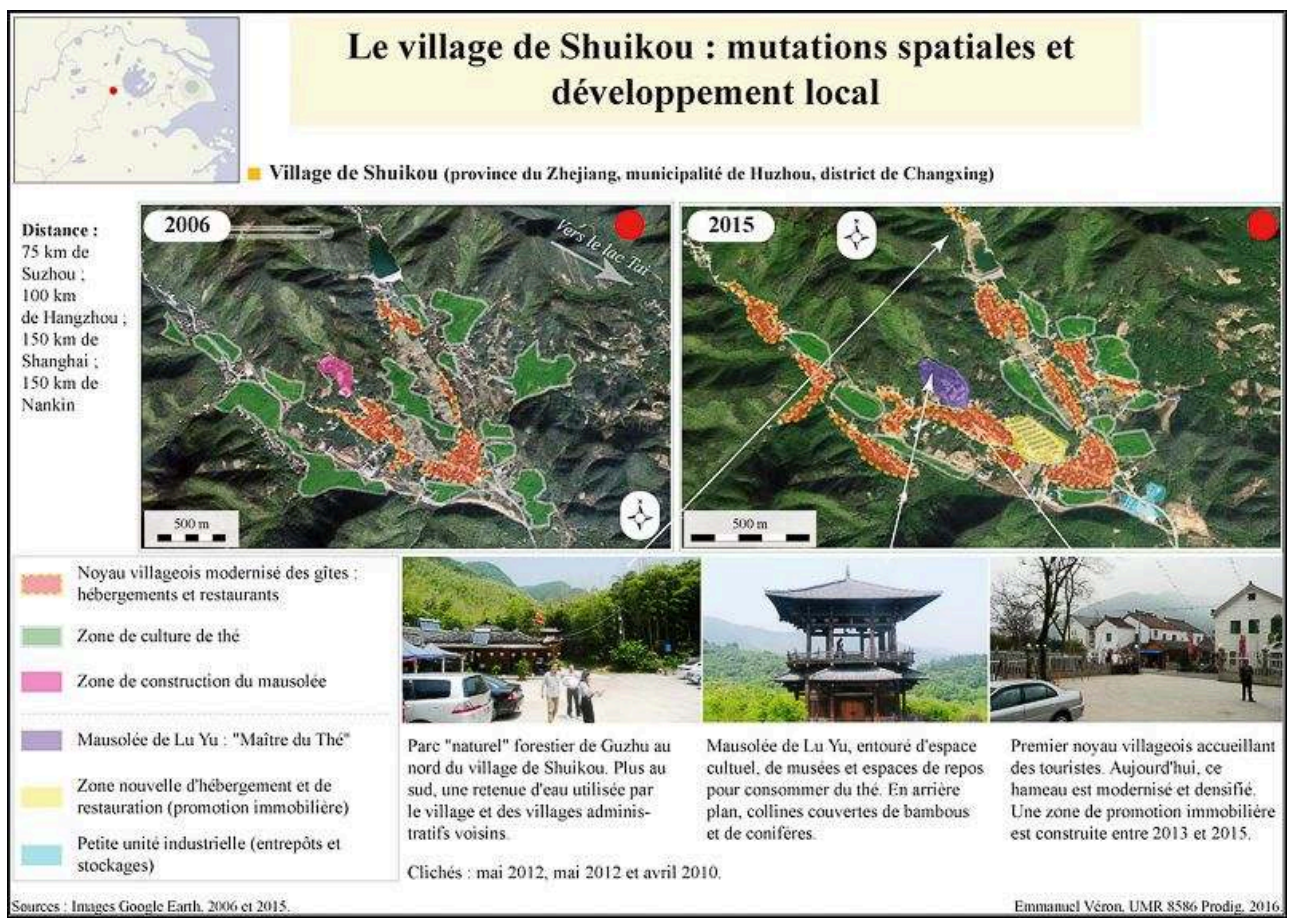

Source : images Google earth 2006 et 2015 ; réalisation : E. Véron, 2016

Une extension de l'espace d'accueil touristique s'opère vers l'est (village de Shuikou) et dans les fonds de vallées adjacents au parc forestier de Guzhu dans le nord. Les gîtes suivent une standardisation organisée par l'association des gîtes des villages de Shuikou et de Guzhu (Shuikou Guzhu cun nongjiale xiehui), ainsi que par les instances de gestion du tourisme du district de Changxing (Bureau de tourisme et Comité du district), le tout supervisé par les autorités de ces mêmes échelons (du village au district). Les prix, la dimension des gîtes, les menus, ainsi que les offres annexes, sont contrôlés en vue d'homogénéiser l'offre. Les recettes touristiques issues des visites du mausolée et du parc forestier sont en partie reversées en parts égales aux différents foyers.

En 2012, il y a environ 400 fermes $^{9}$ proposant chambres d'hôtes ou gîtes et des formules de restauration. Les deux villages de Guzhu et de Shuikou comptent environ 2000 emplois directs dans le tourisme (hébergements, restaurations, guides, entretiens et commerçants). Le bureau de tourisme de Changxing enregistre le chiffre de 2,5 millions de touristes pour l'année 2015. L'activité touristique a produit une recette de plus de 450 millions de yuans. Le budget moyen par jour et par touriste est de 60 à 100 yuans (restauration et hébergement).

Les recettes touristiques assurent un revenu mensuel moyen par paysan de 17600 yuans $^{10}$. Les recettes touristiques ont permis au gouvernement local d'investir dans la modernisation du village. Au total, il y a environ 63 millions de yuans d'investissements dans les infrastructures routières, les équipements (réseaux, chauffeeau), l'agriculture, la sylviculture et une station d'épuration ainsi qu'une usine d'eau minérale. Par ailleurs, les recettes du tourisme permettent la restauration et l'extension des espaces cultuels de la vallée d'une part et un entretien des terroirs de thé environnant les villages naturels d'autre part. La création d'un label local de thé a 
permis en complément de l'image touristique du village une augmentation forte du prix de vente du kilogramme de thé.

Illustration 6 - Modernisation rurale et nouvelle morphologie du bâti

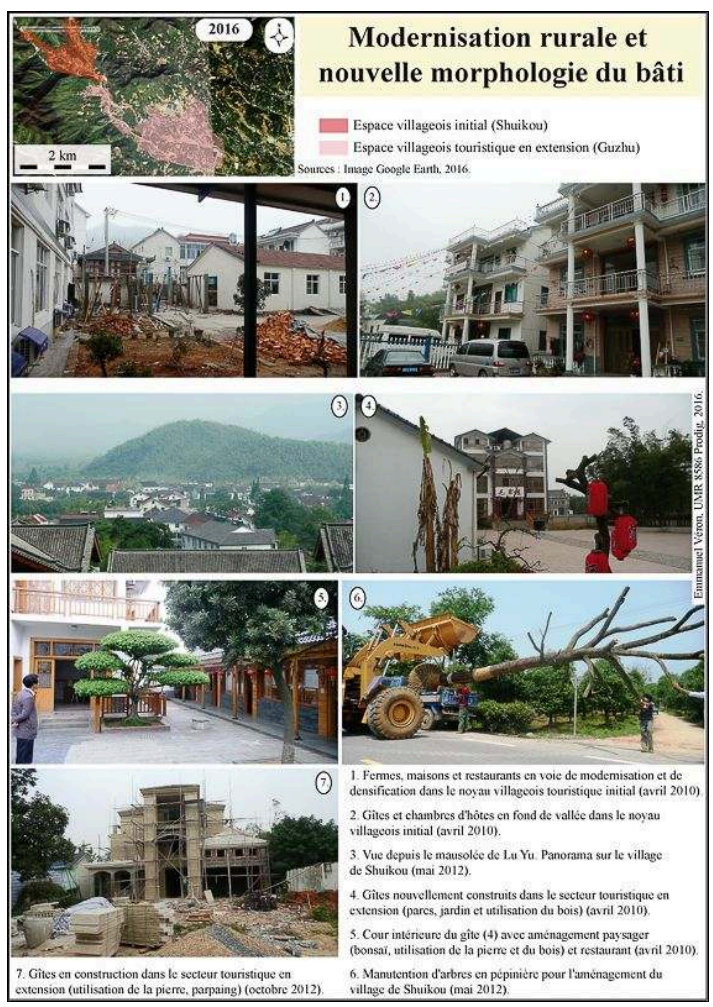

Auteur : E. Véron, 2016.

Les stratégies d'insertion dans le processus touristique s'étalent sur environ deux décennies et connaissent une assez grande hétérogénéité. En effet, le secteur touristique initial rassemblait au début des années 2000 environ 70 à 80 foyers dont les activités marchandes sont complémentaires d'activités agricoles, notamment la culture du thé et du riz. Après 2000, le nombre de foyers augmente de manière importante, passant de 90 à 350 foyers ruraux, débordant sur le village de Shuikou plus loin dans la vallée (environ $5 \mathrm{~km}$ ).

Les populations rurales sont segmentées entre trois catégories de revenus: revenus agricoles majoritaires, revenus non agricoles et revenus touristiques majoritaires. La première catégorie regroupe environ $40 \%$ des plus de 50 ans, tandis que la dernière catégorie est composée par plus de $50 \%$ des moins de $50 \mathrm{ans}^{11}$. La part des revenus non agricoles (en dehors du tourisme) n'a cessé de diminuer depuis 2000. Cela rend compte de la diversification des revenus non agricoles dans les campagnes d'une part, et de la montée en puissance du secteur tertiaire, en particulier du tourisme, d'autre part. La segmentation générationnelle témoigne aussi de comportements et de capacités à entreprendre et investir dans un domaine nouveau. Le lien à la terre n'est pas abandonné par les générations les plus âgées, tandis que les générations les plus jeunes possèdent des ressorts plus importants, en partie acquis avec la pluriactivité dans le village, dans le bourg voire dans les villes. 


\section{L'exemple d'une famille rurale devenue prestataire de services touristiques : le gîte du Jardin des parfums}

Parmi les ruraux locaux avec lesquels nous avons pu nous entretenir figure M. Zhang, responsable d'un gîte majeur du site touristique, mais situé en périphérie du village et du mausolée (éloigné d'environ $5 \mathrm{~km}$ ). M. Zhang, né en 1971, est membre du PCC depuis 2005 et il est responsable de la propagande à la cellule du Parti du village de Shuikou. Il entretient des liens étroits avec les membres du comité de villageois de Shuikou, ainsi qu'avec le comité de villageois voisin de Guzhu et de sa cellule du Parti. Par ailleurs, grâce à son statut, son réseau de relations est assez étendu jusqu'au gouvernement du canton, du district et de la municipalité. Il possède un hukou agricole (nongye hukou), mais n'exerce pas directement d'activité agricole. C'est au début des années 2000 que M. Zhang entreprend son activité marchande d'accueil à la ferme. Il propose une formule simple de restauration à une clientèle régionale ${ }^{12}$.

Les changements du milieu des années 2000 ont stimulé les projets d'investissements conséquents dans le tourisme. En 2008, M. Zhang crée son gîte de Qingxiangyuan ( «le Jardin des parfums »). Il investit dans un nouveau restaurant et dans la construction d'un bâtiment nouveau d'hébergement (15 chambres). En 2012, M. Zhang réalisait un chiffre d'affaires d'environ 100000 yuans par an, employait 5 personnes hors du cercle familial et 8 membres de sa famille (lui compris). Le restaurant emploie 4 personnes en cuisine. Une personne est employée pour l'entretien du gîte. La femme et les enfants de M. Zhang participent à la gestion et l'entretien du site.

Illustration 7- Les prestataires de services touristiques : l'exemple du gîte de Quingxiangyuan

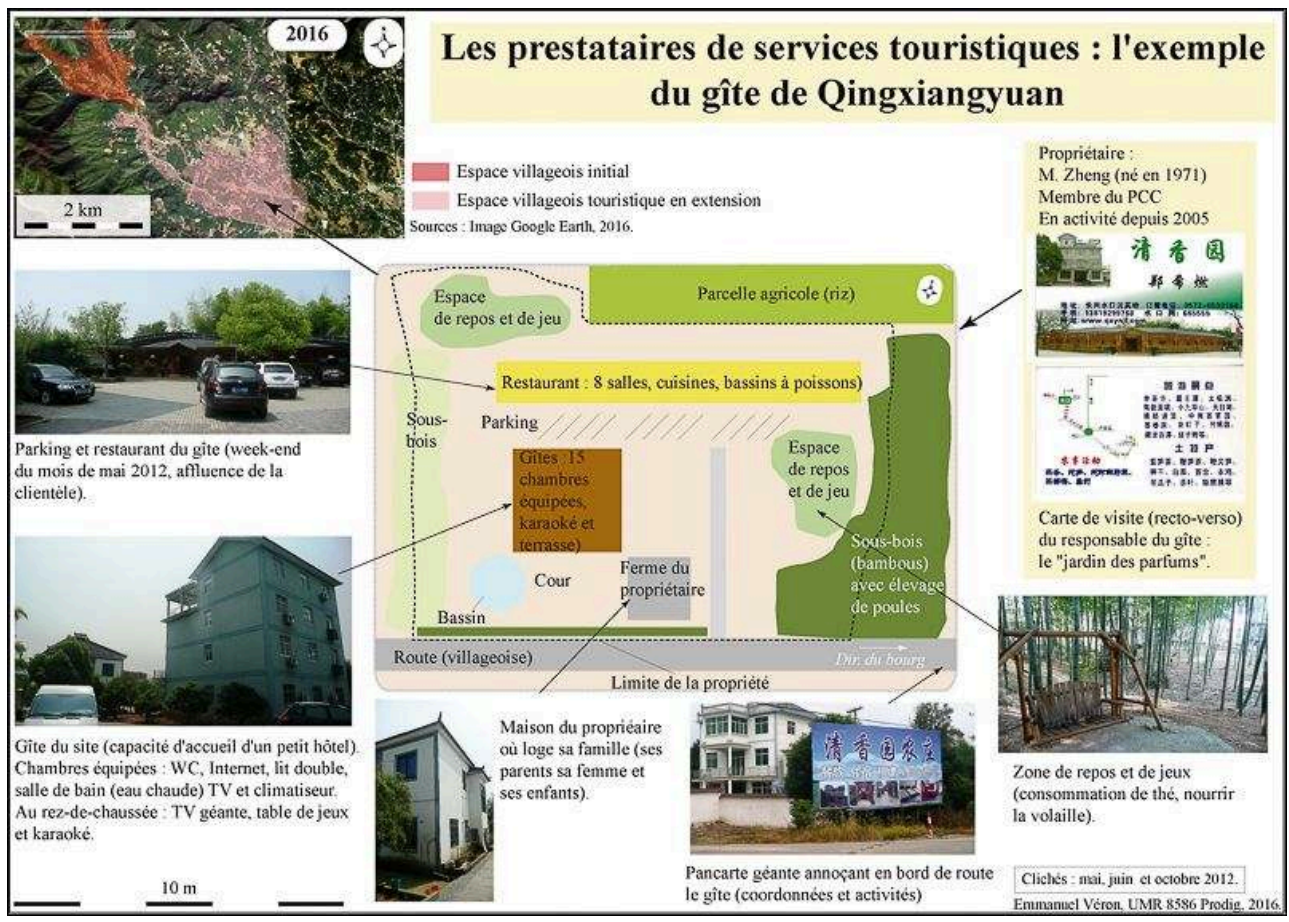

Auteur : E. Véron, 2016.

$\mathrm{Du}$ fait de son réseau de relations (Thireau et Hua, 2002, p. 115), son gîte accumule rapidement les certifications locales, régionales et provinciales lui assurant une 
visibilité majeure chez les voyagistes. Le gîte est successivement référencé comme gîte modèle du district de Changxing, de Huzhou puis de la province du Zhejiang. Le gîte figure parmi les 10 gîtes modèles de Huzhou et de Changxing. Chacune de ces certifications est décidée par les gouvernements locaux et les cellules du PCC à chaque échelon.

Par ailleurs, M. Zhang via l'association des gîtes de Shuikou et le comité de villageois ainsi que le bureau de tourisme du district de Changxing, développe ses liens avec des voyagistes de Shanghai. Ces relations lui assurent un taux de remplissage important, composé à $70 \%$ de clients de la métropole de Shanghai. La formule est de 150 yuans par jour comprenant l'hébergement et la restauration. Ses investissements sont rentabilisés au bout de deux années d'activités et lui permettent d'acheter un second véhicule, et de constituer une épargne pour les études de ses filles (projet d'études à l'étranger). Il peut restaurer et équiper sa maison, où logent ses parents. Cette démarche d'entrepreneur amène $M$. Zhang à être en relation avec plusieurs entreprises locales (constructions et équipements), mais également extrarégionales (paysagisme et architecture). M. Zhang démarche des entreprises dans le bâtiment du bourg voisin afin de construire le gîte, le restaurant, le parking et les éléments annexes (marre pour la pêche à la ligne, aires de jeux). Par ailleurs, afin d'obtenir des certifications, M. Zhang fait intervenir en 2008, une société d'architecture et de paysagiste pour dessiner le gîte et le restaurant ${ }^{13}$. Ces derniers doivent suivre des normes de construction et de sécurité. Ces normes sont généralisées à l'échelle du village par le bureau hygiène et sécurité du district de Changxing.

Le nom du gîte, l'utilisation des matériaux comme le bois (bambous et résineux), ainsi que la morphologie générale du site témoignent d'une certaine modernité et du statut $\mathrm{du}$ responsable du site. Les préceptes classiques du jardin ${ }^{14}$ et de l'aménagement paysager sont mobilisés afin de bonifier l'apparence du lieu et par extension la réputation du propriétaire. Les éléments de l'eau, du bois, des champs, du relief (shui, lin, tian, shan) sont systématiquement utilisés conduisant parfois à une surenchère morphologique. L'utilisation de rochers, du bois et de la végétation, des perspectives (Parkes, 2004) témoignent de la sensibilité des prestataires à rendre compte d'un certain niveau culturel et intellectuel en mettant en scène des règles du fengshui (géomancie). La correspondance entre les montagnes et les pierres a un sens profond. Les pierres sont comme les montagnes, animées par des forces similaires tout en suggérant monts et sommets.

Le style des détails architecturaux du restaurant rappelle ceux des édifices Qing. Tandis que l'architecture du gîte est totalement inédite, basée sur la morphologie de villas construites en parpaings avec une façade en carrelage.

M. Zhang est en lien avec des fournisseurs locaux et ou non pour alimenter son restaurant sur l'ensemble de l'année. Le gîte est moins utilisé entre les mois d'octobre et de mars. Le contrat de travail des personnels en cuisine (population locale) comprend la période de basse intensité et leur permet de diversifier leur revenu dans d'autres activités (construction, chauffeur etc.). Il s'approvisionne en céréales, légumes et fruits d'une part, viandes blanches et rouges d'autre part auprès de paysans locaux) et complète avec des fournisseurs occasionnels inscrits dans des logiques de filières. Ainsi, les logiques locales et de filières coexistent afin de constituer un menu spécifique et quasi homogène à l'ensemble des différents gîtes du site. Par conséquent, la 
gastronomie, au cœur des motivations de consommation touristique des citadins, est calibrée, spécialisée et systématisée.

M. Zhang est au cœur de la réussite de son entreprise. Cet entrepreneur est en lien constant avec les voyagistes de Shanghai, de Hangzhou et de Huzhou. Il a développé son offre via les sites Internet de voyagistes (à l'instar de Ctrip, TripAdvisor ou Mafengwo). Il entretient une forme de sociabilité avec la clientèle citadine (variée) à travers la relation marchande. Il conserve les coordonnées des clients (cartes de visite, mails, numéros de téléphone) et propose des formules adaptées pour les clients réguliers. La recette touristique est hétérogène et suit une saisonnalité étalée entre les mois de mars et d'octobre. Par conséquent, M. Zhang réalise plus de $50 \%$ de son chiffre d'affaires en moins de 6 mois (avril-septembre). Durant la période creuse, le responsable entretient ses revenus par l'organisation de repas pour des officiels, des chefs d'entreprise et des offres diverses proposées à son réseau de relations. En cela, le gite rural n'est plus seulement un lieu de sociabilité pour les touristes citadins, mais également un objet de sociabilité pour les élites rurales locales trouvant dans le restaurant modernisé un espace renouvelé des jeux de face et de mise en valeur de leur réseau de relations. À travers la modernisation des lieux touristiques, une certaine gentry rurale (composée de cadres du Parti, de responsables locaux et de chefs d'entreprises rurales) tisse une structure de sociabilité, de relations et d'affaires. La construction d'un lien social renouvelé (Bonerandi et Hochedez, 2007) est permise par la (ré)activation des réseaux de relations (Ying et Zhou, 2007) donnant une identité professionnelle du prestataire de service touristique proche des élites rurales.

M. Zhang projette d'élargir son offre touristique par l'aménagement d'une parcelle agricole destinée à la cueillette de fraises durant le printemps d'une part et la construction d'une petite ferme d'autre part. Les parcelles pourront être louées aux citadins de manières ponctuelles ou mensuelles. Il envisage aussi de moderniser son gîte par de nouveaux équipements de jeux et de divertissements (changement des téléviseurs, tables de jeux, nouveaux matériels de karaoké). Le renouvellement de l'offre est au cœur du projet de l'entrepreneur.

\section{Conclusion : le gîte comme vecteur de diversification des revenus, de sociabilités renouvelées et de nouvelles ruralités}

Les activités tertiaires liées au tourisme croissent de manière importante dans les espaces ruraux du delta et constituent un nouvel élément dans les créations d'emplois et d'intégration ville-campagne. Alors que de nombreux projets dans les années 1990 avaient exclu, dédommagé et relogé les communautés rurales des lieux (Ryan et al., 2009), le tourisme dans les campagnes à travers le projet de nongjiale permet aux ruraux de diversifier leurs revenus et d'être des acteurs de premier plan dans le processus touristique. Ce rôle est cependant limité à l'activité marchande de l'hébergement, de la restauration et de la vente de produits.

50 L'intensité des revenus de l'agriculture est inversement proportionnelle à ceux de l'activité touristique. La valeur des productions agricoles est bien souvent inférieure aux recettes touristiques à l'échelle d'une famille. Pour autant, la dimension agricole participant à la mise en scène du gîte et du restaurant n'est pas abandonnée. 
51 La modernisation du village et l'amélioration des conditions de vie des communautés rurales (Bray, 2013) sont aussi marquées par l'apport de nouvelles technologies qui n'étaient auparavant possible de trouver que dans les villes (eau chaude, système d'égouts, climatiseur, gaz, câble digital, Internet, etc.). Une construction résidentielle stimulée par la maison individuelle accolée à un gîte et un restaurant poursuit le renouvellement de la morphologie des campagnes, contrastant avec l'image des campagnes chinoises, parents pauvres du développement des trente dernières années.

Ce développement local par le tourisme de communautés rurales tirant l'essentiel de leur revenu en dehors de l'agriculture et d'activités industrielles offre une remarquable illustration des mutations sociales et touristiques. La montée en puissance du secteur non agricole segmente les familles, l'emploi et les revenus. La répartition des richesses entre communautés rurales, acteurs privés et gouvernements locaux produit un nouveau mode de gouvernance.

À la faveur de l'augmentation du capital (économique et relationnel) de nouvelles élites rurales émergent dont les propriétés sont autant de nouveaux espaces de sociabilité pour les ruraux eux-mêmes dans une logique multiscalaire (du village au district rural). Le restaurant et les jardins aménagés à la façon d'anciennes demeures deviennent des repères sociaux pour la nouvelle gentry rurale.

Ces élites locales sont de manière récurrente, à l'origine de la trajectoire touristique des villages, renouvelant les espaces de sociabilité et de pouvoir. Le tourisme dans les villages participe à la refondation de la communauté autour d'un projet. La notion de cunfeng (tendance ou trajectoire villageoise) développée par Yan (2002, p. 148) prend ici tout son sens. Le tourisme produit un effet de lieu particulier au village, dont les élites locales jouissent pour conduire leurs affaires et étendre leurs réseaux de relations à travers le district, la municipalité voire la province.

\section{BIBLIOGRAPHY}

Attané I., 2002. Un demi-siècle de socialisme chinois : heurs et malheurs de la famille paysanne. In Attané I. (dir.), 2002, La Chine au seuil du XXIe siècle. Questions de population, questions de société, Paris, INED, 602 p., chapitre 13, p. 355-388.

Aubert C., 2005. Le devenir de l'économie paysanne en Chine. Revue Tiers Monde, Paris, PUF, $\mathrm{n}^{\circ} 183$, p. 491-515.

Beaumont N., Dredge D., 2010. Local tourism governance: A comparison of three network approaches. Journal of Sustainable Tourism, 18(1), p. 7-28.

Bramwell B., Lane B., 2011. Critical research on the governance of tourism and sustainability. Journal of Sustainable Tourism, 19(4-5), p. 411-421.

Bray D., 2013. L'urbanisme à la campagne. Perspectives chinoises, vol. 3, p. 57-67.

Charon P., 2013. Exégèse des institutions villageoises : un échelon de l'autonomie ? SinoPolis. http://sinopolis.hypotheses.org/425 
Church A., Coles T., 2006. Tourism, power and space. Routledge.

Démurger S., Xu H., 2011. Return migrants: The rise of new entrepreneurs in rural China. World Development, $\mathrm{n}^{\circ} 10$, p. 1847-1861.

Hall C. M., 2003. Politics and place: An analysis of power in tourism communities. Tourism in destination communities, p. 99-114.

He J. et al., 2004. Rural Tourism in China. A Case Study of Nongjiale in the Chengdu Metropolitan Area. Mountain Research and Development, $\mathrm{n}^{\circ} 3$, p. 260-262.

Hu M., 2007. Xiangcun minxiu jing yingguan li huxin ziyuan fenxi, [Analyse des ressources centrales dans la gestion de l'hébergement rural]. Beijing, China, Lüyou xuekan, Tourism Tribune, $\mathrm{n}^{\circ}$ 9, p. 64-69.

Hu M., 2009. Wo guo xiangcun lüyou zhuanye hezuo zuzhi de fazhan he zhuanxing-jianlun xiangcun lüyou fazhan moshi de shengji [Changements et développement de l'organisation des coopérations spécialisées dans le tourisme rural en Chine - amélioration du modèle de développement du tourisme rural]. Beijing, China, Lüyou xuekan, Tourism Tribune, $\mathrm{n}^{\circ}$ 2, p. 70-74.

Parkes G., 2004. La Pensée des Rochers ? La Vie des Pierres. Réflexions sur une passion chinoise. Diogène, $\mathrm{n}^{\circ}$ 207, p. 95-111.

Ren Y., 2011. La coopérative en participation : une nouvelle forme de l'économie collective en Chine rurale. Thèse de doctorat en économie, sous la direction de Pairault T., EHESS, 334 p.

Rodriguez G. et al., 2011. From Farm to Rural Hostel: New Opportunities and Challenges Associated with Tourism Expansion in Daxi, a Village in Anji County, Zhejiang, China. Sustainabily, $\mathrm{n}^{\circ} 3$, p. 306-321.

Ryan C. et al., 2009. Community Participation and Social Impacts of Tourism. In Ryan C. et Gu Huimin, Tourism in China: Destination, Cultures and Communities, Routledge, London, $400 \mathrm{p}$, p. 239-258.

Sanjuan T., 2011. Les nouvelles relations ville-campagne en Chine aujourd'hui. In Guibert M. et Jean Y. (dir.), Dynamiques des espaces ruraux dans le monde, Paris, Armand Colin, p. 217-228.

Shao Q., 2007. Cujin xin nongcun jianshe yu fazhan xiangcun lüyou, [Développer le tourisme rural et promouvoir la construction des nouvelles campagnes]. Beijing, China, Qiushi, n 1, p. 42-44.

Su B., 2011. Rural tourism in China, Tourism Management, p. 1-4.

Thireau I., Hua L., 2002. À l'ombre des commerces en bordure de route. Études rurales, n 161-162, p. 109-127.

Véron E., 2013. Les espaces ruraux touristiques dans le delta du Yangzi, entre intégration villecampagne et développement rural. EchoGéo [En ligne], 26 | 2013, mis en ligne le 19 décembre 2013. http://journals.openedition.org/echogeo/13607 ; DOI : 10.4000/echogeo.13607

Véron E., 2016. Les espaces ruraux à l'heure du tourisme citadin. L'exemple du delta du Yangzi - Chine. Thèse de doctorat sous la direction de Thierry Sanjuan, Université Paris 1 Panthéon-Sorbonne, $696 \mathrm{p}$.

Véron E., 2016. Nouvelles politiques de développement rural en Chine : vers de nouvelles normes d'aménagement dans les campagnes du delta du Yangzi. L'Information géographique, nº 1, p. 55-71. Yan Y., 2002. Planning birth: Changes in Fertility Culture in a Chinese Village. Études rurales, $\mathrm{n}^{\circ}$ 161-162, p. 129-152. 
Yang W., 2005. Réformes, ajustements structurels et revenu rural en Chine. Perspectives chinoises, $\mathrm{n}^{\circ}$ 92, 11 p. http://perspectiveschinoises.revues.org/930

Ying T., Zhou Y., 2007. Community, governments and external capitals in China's rural cultural tourism: A comparative study of two adjacent villages. Tourism Management, $\mathrm{n}^{\circ} 1$, p. 96-107.

Zheng Q., Zhong L., 2004. Shequ canyu xiangcun lüyou fazhan moshi de taolun, [Une discussion sur le modèle de développement de communautés évoluant dans le tourisme rural], Beijing, China. Lüyou xuekan, Tourism Tribu.

\section{NOTES}

1. http://www.chinadaily.com.cn/business/2017-04/11/content_28880107.htm. Ces chiffres avancés par les autorités centrales sont à prendre avec précaution, tant il est difficile de vérifier la véracité de tels volumes, en particulier dans un contexte politique autoritaire. 100 yuans sont l'équivalent de 11,5 à $12,5 €$ pour la période d'étude.

2. Ce travail est issu d'une thèse de doctorat en géographie sur le tourisme dans les espaces ruraux du delta du Yangzi sous la direction de Thierry Sanjuan, Université Paris 1 Panthéon Sorbonne.

3. Seconde instance après la branche du parti, le comité de villageois, composé de trois à sept personnes élues par les habitants, fonctionne en institution autonome et doit trouver lui-même l'argent pour le fonctionnement du village.

4. La culture patriarcale est au centre de l'organisation sociale traditionnelle. Ceci perdure encore aujourd'hui. Le développement touristique d'un gite est avant tout un investissement pragmatique de la famille où chaque membre joue un rôle précis. Les relations entre les différents membres d'une famille sont matérialisées par une entraide des enfants envers les parents et réciproquement (Attané, 2002).

5. Le nom de nongjiale est une marque désignant l'activité marchande touristique de foyers paysans. Ce n'est pas un label institutionnalisé. Mais la diffusion à travers l'ensemble du territoire chinois a permis le développement du tourisme à la campagne par une instrumentalisation de ce nom, afin de permettre aux citadins de distinguer un foyer paysan proposant l'accueil touristique d'une ferme paysanne lambda. Ce mot chinois correspond pleinement au gîte.

6. Entretiens en mai 2012 avec M. Zhang, paysan dans le Zhejiang (village de Shuikou), dont la ferme est équipée pour l'accueil des touristes.

7. Le village est parfois appelé le village de Shanghai (Shanghai cun) tant le tourisme est dominé par une clientèle shanghaienne, plutôt âgée. http://sh.eastday.com/m/20160214/ u1ai9217744.html

8. Madame Zhu est américaine d'origine chinoise. Elle l'une des responsables de la société Seven cups, basée à Tucson. Elle fait également partie de l'Institut pour la culture du thé chinois.

9. Le site comptait moins d'une centaine de gîtes au début des années 2000 (Hu, 2009).

10. http://www.shuikoudq.com/about/?1197.html. Ces chiffres ont été également donnés dans le cadre d'entretiens avec les responsables de la cellule du Parti du village, sans pour autant faire l'objet de vérifications possibles par le croisement avec d'autres données.

11. Une dizaine d'entretiens avec les responsables du comité de villageois de Guzhu (mai et octobre 2012).

12. Pour développer un gîte, il faut obtenir l'autorisation du Bureau de la terre (yongdi ju), afin de protéger les terres cultivables.

13. Des entreprises privées à l'instar de Baijie Gongshe ou de Lingyu ont largement contribué à la transformation morphologique et architecturale des lieux. Ces deux entreprises ont remporté 
des projets d'architecture et de paysagisme dans les villages de Shuikou et de Guzhu, mais également dans une large partie des provinces chinoises. Ces entreprises sont spécialisées dans l'aménagement touristique des lieux à travers des projets de promotions immobilières et paysagères.

14. Le traité des jardins (Yuanye) date de la fin de la dynastie Ming (publié en 1634 par Ji Cheng).

\section{ABSTRACTS}

This article examines the forms of rural governance in the Yangtze River Delta megalopolis with regard to rural tourism. We study the role of communities and their integration into a new economic activity trough tourism services. These activities have led rural communities to reorganize their work, enrich their homes and morphologically transform villages. The study of two villages within the Yangtze delta illustrates local development through tourism and the evolution of rural people who have become tourist service providers.

Cet article analyse les formes de gouvernance rurale de la mégalopole du delta du Yangzi au regard du tourisme dans l'espace rural. Nous étudions le rôle des communautés et leur insertion dans une nouvelle activité économique, celle de services touristiques. Ces activités ont conduit des communautés rurales à une réorganisation du travail, un enrichissement des foyers et à une transformation morphologique des villages. L'examen de deux villages au sein du delta du Yangzi permet d'illustrer un développement local par le tourisme et l'évolution des ruraux, devenus prestataires de services touristiques.

\section{INDEX}

Mots-clés: gouvernance, tourisme, espace rural, communauté rurale, mégalopole, aménagement, delta du Yangzi

Keywords: governance, tourism, rural space, rural community, megalopolis, planning, Yangzi delta

Subjects: Sur le Champ - Sur le Terrain

\section{AUTHOR}

\section{EMMANUEL VÉRON}

Emmanuel Véron, emmanuel.veron@yahoo.fr, est Docteur en géographie, enseignant-chercheur à l'Inalco (département des Études chinoises) \& Société de Géographie et associé à l'équipe ASIEs et à l'UMR Prodig. Il a publié récemment :

- Véron E., 2018. La France en marche sur les nouvelles routes de la soie ?, The Conversation.

- Véron E., 2018. La diplomatie du pourtour de la Chine : "bon voisinage" en Asie ou hégémonie chinoise ? In Richard Y. et Mareï N. (dir.), Dictionnaire des intégrations régionales, Atlande, à paraître. 
- Véron E., 2018. Les routes énergétiques de la soie et la diplomatie du voisinage chinois. S'engager par la plume, Anaj-IHEDN, à paraître. 